\title{
Eating disorders, pregnancy, and the postpartum period: Findings from the Norwegian Mother and Child Cohort Study $(\mathrm{MoBa})$
}

\author{
Hunna J. Watson ${ }^{1,2,3,4}$, Leila Torgersen ${ }^{5}$, Stephanie Zerwas ${ }^{1}$, Ted Reichborn-Kjennerud ${ }^{6,7}$, \\ Cecilie Knoph ${ }^{6}$, Camilla Stoltenberg ${ }^{8,9}$, Anna Maria Siega-Riz ${ }^{10,11}$, Ann Von Holle ${ }^{1}$, \\ Robert M. Hamer ${ }^{1,12}$, Helle Margrete Meltzer ${ }^{13}$, Elizabeth H. Ferguson ${ }^{1,11}$, \\ Margaretha Haugen ${ }^{14}$, Per Magnus ${ }^{8}$, Rebecca Kuhns ${ }^{1}$ and Cynthia M. Bulik ${ }^{1,11,15}$ \\ 1) Department of Psychiatry, University of North Carolina at Chapel Hill, United States \\ 2) Eating Disorders Program, Child and Adolescent Health Service, Department of Health in Western Australia, \\ Perth, Australia \\ 3) School of Paediatrics and Child Health, The University of Western Australia, Perth, Australia \\ 4) School of Psychology and Speech Pathology, Curtin University, Perth, Australia \\ 5) Division of Mental Health, Norwegian Institute of Public Health, Oslo, Norway \\ 6) Division of Mental Health Services, Akershus University Hospital, Oslo, Norway \\ 7) Institute of Clinical Medicine, University of Oslo, Norway \\ 8) Division of Epidemiology, Norwegian Institute of Public Health, Oslo, Norway \\ 9) Department of Global Public Health and Primary Care, University of Bergen, Bergen, Norway \\ 10) Department of Epidemiology, University of North Carolina at Chapel Hill, Chapel Hill, United States \\ 11) Department of Nutrition, University of North Carolina at Chapel Hill, Chapel Hill, United States \\ 12) Department of Biostatistics, University of North Carolina at Chapel Hill, Chapel Hill, United States \\ 13) Division of Environmental Science, Norwegian Institute of Public Health, Oslo, Norway \\ 14) Division of Environmental Medicine, Norwegian Institute of Public Health, Oslo Norway \\ 15) Department of Medical Epidemiology and Biostatistics, Karolinska Institutet, Stockholm, Sweden \\ Correspondence: Cynthia Bulik, E-mail: cynthia bulik@med.unc.edu
}

\begin{abstract}
This review summarizes studies on eating disorders in pregnancy and the postpartum period that have been conducted as part of the broader Norwegian Mother and Child Cohort Study (MoBa). Prior to the 2000s, empirical literature on eating disorders in pregnancy was sparse and consisted mostly of studies in small clinical samples. MoBa has contributed to a new era of research by making population-based and largesample research possible. To date, MoBa has led to 19 eating disorder studies on diverse questions including the prevalence, course, and risk correlates of eating disorders during pregnancy and the postpartum. The associations between eating disorder exposure and pregnancy, birth and obstetric outcomes, and maternal and offspring health and well-being, have also been areas of focus. The findings indicate that eating disorders in pregnancy are relatively common and appear to confer health risks to mother and her child related to sleep, birth outcomes, maternal nutrition, and child feeding and eating.
\end{abstract}

This is an open access article distributed under the Creative Commons Attribution Licence, which permits unrestricted use, distribution, and reproduction in any medium, provided the original work is properly cited.

Eating disorders involve extreme departures from normal eating behavior, such as food restriction, binge eating, and purging, and there are often disturbances in the way shape and weight are experienced (1). The three main types of eating disorders are anorexia nervosa ( $\mathrm{AN})$, bulimia nervosa $(\mathrm{BN})$, and binge eating disorder (BED), which $8.7 \%$ of Norwegian women will experience over the lifetime, and at any one time afflict 3.8\% (2). Eating disorders may be tremendously physically damaging and life-threatening, causing osteoporosis, dental erosion, gastrointestinal problems, kidney damage, and cardiac abnormalities, which combined with a higher suicide risk, result in elevated mortality $(3,4)$. Comorbid depression and anxiety and social and occupational impairments are common (5). Despite their substantial physical, psychological, and social toll, the intersection between eating disorders and pregnancy has been poorly understood.
Scientific inquiry into eating disorders in pregnancy prior to the 2000 s comprised a small collection of clinical studies, subject to significant selection bias, and a lack of population-based research. Topics examined were reasonably diverse and included delayed detection of pregnancy in AN, course of eating disorders in pregnancy, sudden maternal death in pregnancy, breastfeeding, birth outcomes, and prepartum and postpartum adjustment. The evidence base, however, was confusing and lacked integration, for instance, some studies hinted at unfavorable birth and early childhood outcomes $(6,7)$ while others did not $(8)$. The science at that point was unable to answer critical questions that could only reasonably be answered through population-based research, such as the prevalence of eating disorders during pregnancy and impact on birth outcomes. Two advancements were needed; a population-based sampling frame to investigate eating 
disorders in general and not only among the 40\% (5) who seek treatment, and large cohorts, to achieve adequate statistical power.

Crucial scientific insight about eating disorders in pregnancy has since emerged through The Norwegian Mother and Child Cohort Study (MoBa) coupled with other international birth cohorts $(9,10)$. Key comparative strengths that distinguish the $\mathrm{MoBa}$ analyses are the availability of a large overall sample $(N>100,000$ pregnancies), a population-based cohort design, nationally representative recruitment - which over time grew to 50 of 52 hospitals in Norway (only facilities with at least 100 births annually were targeted), and the breadth of exposures assessed. First, a brief summary of the MoBa design is given, next, the corpus of research (19 studies to-date) is summarized and in each section, MoBa findings are integrated with existing research, theory, and broader public health literature. Table 1 accompanies the text, giving specific details of each study's findings. The paper concludes with limitations and challenges of the research, research directions, and a summary conclusion.

\section{The MoBa STUdy}

MoBa (11) is a population-based pregnancy cohort study established to identify causes of disease to inform prevention. The study is conducted by the Norwegian Institute of Public Health. Recruitment began in 1999, and the target population was all women giving birth in Norway. Women who attended routine ultrasound testing in week 17 to 18 of pregnancy received a postal invitation to participate in the study, which included the informed consent form and the first questionnaire survey. The sampling unit for the study is pregnancy, meaning that women may participate with more than one pregnancy. Recruitment started with a single hospital and grew to include 50 of 52 hospitals with maternity facilities in Norway from 2005-2008. The women consented to participation in $40.6 \%$ of the pregnancies. The cohort now includes 114,500 children, 95,200 mothers and 75,200 fathers. Questionnaires have been administered during pregnancy, at 6 months, 18 months, 3 years, 5 years, 7 years, and 8 years after birth, and a biobank is available.

The questionnaire that pregnant women completed during pregnancy included self-report items on eating disorders developed for MoBa. The items were based on Diagnostic and Statistical Manual (DSM-IV-TR) nomenclature (12) and allowed caseness for $\mathrm{AN}, \mathrm{BN}$, $\mathrm{BED}$, and eating disorders not otherwise specifiedpurging type (EDNOS-P) to be evaluated six months prior to and during pregnancy. EDNOS-P refers to the presence of recurrent purging in the absence of binge eating. Caseness was established through self-report without interview or clinical evaluation.

\section{OTHER POPULATION-BASED RESEARCH}

The Avon Longitudinal Study of Parents and Children (ALSPAC) in the United Kingdom includes similar questions to MoBa, but has a smaller sample $(\sim 14,000$ mothers were enrolled). ALSPAC has made important contributions to the literature on eating disorders and pregnancy, and will be referred to where relevant. ALSPAC limitations are that eating disorder diagnoses did not follow accepted classification (i.e., Diagnostic and Statistical Manual [DSM], International Classification of Disease) and only ' $\mathrm{AN}$ ' and 'BN' were considered.

\section{EPIDEMIOLOGY}

\section{Prevalence}

MoBa research (13) was the first to yield estimates of the prevalence of eating disorders in pregnancy using Diagnostic and Statistical Manual criteria (14). The $\sim 5 \%$ observed prevalence compares with general population rates (2). The prevalence estimates were established as valid in a subsequent internal validity study $(5,15)$. An ALSPAC paper $(N=12,254)$ (16) published at about the same time as the MoBa paper on prevalence considered recent and past $\mathrm{AN}$ and $\mathrm{BN}$ and found a 12 week pregnancy prevalence of $0.05 \%$ and $0.4 \%$, respectively, and lifetime prevalence of either $\mathrm{AN}, \mathrm{BN}$, or both of $3.2 \%$. A recent prospective study $(N=739)(17)$ in a prenatal clinic used a selfreport diagnostic tool at three months gestation and found an overall point prevalence of $7.5 \%(0.5 \% \mathrm{AN}$, $0.1 \%$ BN, $1.8 \%$ BED, $0.1 \%$ EDNOS-P, $5 \%$ other EDNOS). As presupposed, other EDNOS presentations increased the overall prevalence estimate. The estimates regarding eating disorder subtypes are similar.

\section{Course of Illness}

MoBa research has yielded insight into the trajectory of illness from pre-pregnancy to during pregnancy and postpartum $(13,15,18)$. Similar to the MoBa findings, remission from $\mathrm{BN}$ in pregnancy has been noted in previous literature $(8,19)$, as have incident eating disorders (20). Clinical narratives and some scientific data $(8,19)$ have suggested that while some women with $\mathrm{BN}$ may cease binge eating and purging during pregnancy and stay well postpartum, in other women there is a "spike" in disordered eating postpartum. Women with eating disorders in pregnancy who become well postpartum report no longer having time to binge eat, not wanting their children to imitate the disordered eating behaviors, and new meaning in life that reduces focus on weight and shape $(8,21)$. Neurobiological or other explanations are not within reach of self-report methodology. Conversely, after delivery, some women may be struggling to accept or manage pregnancyrelated weight gain, setting up a risk pathway to further dieting and disordered eating and putting mother and child at risk of undesirable health outcomes. Postpartum depression screening is widely accepted and commonplace, dialogue on the importance, methods, barriers, and follow-up care on postpartum detection of eating disorders is needed. 
Table 1. MoBa studies on eating disorders in pregnancy.

\begin{tabular}{ll}
\hline $\begin{array}{l}\text { Authors: year } \\
\text { (reference \#) }\end{array}$ & Research area \\
\hline Bulik, Von Holle, & $\begin{array}{l}\text { Prevalence and } \\
\text {.., Reichborn- }\end{array}$ \\
Kjennerud: & $\begin{array}{l}\text { course of illness of } \\
\text { eating disorders }\end{array}$ \\
2007 (13) & during pregnancy
\end{tabular}

Study highlights

One in 21 pregnant women had some type of eating disorder $(0.2 \% \mathrm{BN}, 4.8 \% \mathrm{BED}$,

$<0.1 \%$ EDNOS-P). In the six months prior to pregnancy, $0.1 \%$ reported $\mathrm{AN}$; EDNOS-P tended to remit during pregnancy (78\%). BED typically continued $(61 \%)$. BN outcomes varied among remitted $(34 \%)$, partially remitted $(34 \%)$, or continued course (29\%). Incident cases of BED during pregnancy were common (2.1\%). AN was not assessed during pregnancy because the weight criterion was judged as unable to be reliably evaluated using self-report given weight gain (i.e., fetus, amniotic fluid, placenta). For BN, self-induced vomiting for weight loss was differentiated from pregnancy-induced nausea in survey questions.

\begin{tabular}{ll}
\hline Watson, Von Holle, & Validation study on \\
prevalence and \\
$2012(15)$ & $\begin{array}{l}\text { course of illness of } \\
\text { eating disorders } \\
\text { during pregnancy }\end{array}$ \\
\hline Knoph Berg, Von & $\begin{array}{l}\text { Course of illness } \\
\text { Holle, }, \ldots, \text { Bulik: }\end{array}$ \\
from pre-pregnancy \\
to13 (18) & to postpartum
\end{tabular}

The original study was based on the cohort approximately halfway into recruitment; this study was conducted when all pregnancies were recruited, and gave evidence of the internal validity of the findings regarding prevalence and course of illness in Bulik et al. (13).

The prevalence of remission from pre-pregnancy AN was 50\% at 18 months postpartum and $59 \%$ at 36 months postpartum. For pre-pregnancy $\mathrm{BN}$, remission prevalence was $39 \%$ at 18 months and $30 \%$ at 36 months, $46 \%$ and $57 \%$ for prepregnancy EDNOS-P, and $45 \%$ and $42 \%$ for pre-pregnancy BED. Many women who had eating disorders pre-pregnancy still experienced them at a clinical level more than two years later.

Knoph Berg, Bulik, Risk correlates for $\quad$ BN during pregnancy was significantly predicted by lifetime exposure to major ..., Reichborn- BN depression, sexual abuse, physical abuse, and smoking and current higher anxiety Kjennerud: and depression symptoms, lower life satisfaction, lower self-esteem, lower $2008(22)$ relationship satisfaction, and higher alcohol use. Incident BN was significantly more likely among those with higher depression and anxiety, lower self-esteem, and lower life satisfaction. Remission of BN in pregnancy was significantly more likely among women with lower anxiety and depression, higher life satisfaction, and higher self-esteem.

Watson, Von Holle, Validation study on The original study was based on the cohort approximately halfway into recruitment; ..., Bulik: risk correlates for BN this study was conducted when all pregnancies were recruited, and gave evidence of In press (24) the internal validity of the findings regarding the associations between psychosocial factors and BN prevalence and course of illness from pre-pregnancy to during pregnancy in Knoph Berg et al (22).

\begin{tabular}{lll}
\hline $\begin{array}{l}\text { Knoph Berg, } \\
\text { Torgersen, ... } \\
\text { Reichborn- }\end{array}$ & $\begin{array}{l}\text { Risk correlates for } \\
\text { Kjennerud: }\end{array}$ & $\begin{array}{l}\text { Fourteen of fifteen psychosocial factors investigated were statistically significantly } \\
\text { associated with incident BED. Remission from BED during pregnancy was } \\
\text { significantly more likely among women who perceived themselves as overweight } \\
\text { prior to pregnancy and placed less emphasis on weight as a measure of their self- } \\
\text { worth. Women who had continuation of BED during pregnancy were less likely to } \\
\text { perceive themselves as overweight prior to pregnancy. }\end{array}$ \\
\hline $\begin{array}{ll}\text { Bulik, Hoffman, } \\
\ldots, \text { Reichborn- }\end{array}$ & $\begin{array}{l}\text { Unplanned } \\
\text { pregnancies in AN }\end{array}$ & $\begin{array}{l}\text { There was a more than two-fold higher rate of unintended pregnancies among } \\
\text { MoBa women with AN compared with referent women. }\end{array}$
\end{tabular}

Kjennerud:

2010 (27)

Bulik, Von Holle, Birth and obstetric ..., Reichborn- outcomes Kjennerud: 2009 (29)

\begin{tabular}{|c|c|c|}
\hline $\begin{array}{l}\text { Bulik, Von Holle, } \\
\ldots, \text { Reichborn- } \\
\text { Kjennerud: } \\
2009 \text { (29) }\end{array}$ & $\begin{array}{l}\text { Birth and obstetric } \\
\text { outcomes }\end{array}$ & $\begin{array}{l}\text { Primary birth outcomes were low birth weight, preterm delivery }(<37 \text { weeks), } \\
\text { small-for-gestational-age, large-for-gestational-age, preeclampsia, Caesarean } \\
\text { section, gestational diabetes, and slow progress. In unadjusted models, women with } \\
\text { recent or acute BED were at a significantly higher risk of large-for-gestational age } \\
\text { babies and Caesarean section, and lower risk for small-for-gestational-age babies. } \\
\text { No differences were observed on primary birth outcomes for AN, BN, or EDNOS- } \\
\text { P. Secondary birth outcomes were anaesthetics during labor, assistance at vaginal } \\
\text { breech presentation, epidural, HELLP, nonvertex presentation, and placenta previa. } \\
\text { In unadjusted models, there was a greater probability of epidural among women } \\
\text { with a recent history of AN and assistance at vaginal breech presentation among } \\
\text { women with recent or current BN, which remained significant after adjustment. In } \\
\text { adjusted models, there was a greater probability of epidural among women with } \\
\text { BED. }\end{array}$ \\
\hline $\begin{array}{l}\text { Bulik, Von Holle, } \\
\ldots, \text { Reichborn- } \\
\text { Kjennerud: } \\
2008(32)\end{array}$ & Sex ratio & $\begin{array}{l}\text { There were non-significant but by magnitude potentially meaningful differences in } \\
\text { sex ratio by eating disorder presence. Risk ratios of proportion male offspring } \\
\text { adjusted for sociodemographic and maternal factors were } 0.89 \text { for AN, } 0.93 \text { for BN, } \\
1.28 \text { for EDNOS-P, and } 1.04 \text { for BED. }\end{array}$ \\
\hline
\end{tabular}




\begin{tabular}{ll}
\hline Swann, Von Holle, & Attitudes to weight \\
.., Bulik: & gain in pregnancy \\
2009 (35) &
\end{tabular}

\begin{tabular}{ll}
\hline Siega-Riz, Von & Gestational weight \\
Holle, .., Bulik: & gain \\
$2011(38)$ &
\end{tabular}
$2011(38)$
Worry about pregnancy-related weight gain was significantly higher in AN, BN, BED, and EDNOS-P than women without eating disorders. Women with BED who were very worried about weight gain had larger gestational weight gain. Referent women with greater worry had higher gestational weight gain, higher infant weights, greater likelihood of a large-for-gestational-age infant, and lower likelihood of a small-for-gestational-age infant.

Women in MoBa with recent or current BN or BED gained significantly more weight than referent women. Women with recent AN gained the most weight throughout pregnancy, although statistical significance could not be tested due to small sample size. Weight gain classifications followed Institute of Medicine guidelines. Referent women: $24 \%$ gained weight inadequately, $26 \%$ gained an adequate amount, and $50 \%$ gained weight excessively. Women with BN and BED: $20 \%$ and $18 \%$ gained inadequately, $20 \%$ and $17 \%$ gained adequately, and $60 \%$ and $65 \%$ gained excessively. Excessive weight gain in BN and BED remained after adjustment for pre-pregnancy body mass index.

Torgersen, Von Pregnancy-related Women with BN purging type and EDNOS-P, but not women with BN non-purging Holle, ..., Bulik: nausea and vomiting type, had significantly higher odds of pregnancy-related vomiting compared with $2008(38)$ referent women. Nausea was more common amongst the BN purging subtype, and there were no differences amongst groups in presence of hyperemesis gravidarum. Women with BED before and during pregnancy had significantly higher overall energy intake, total fat, monounsaturated fat, and saturated fat consumption, and lower intakes of folate, potassium, and vitamin C compared with the referent group. Women with onset of BED during pregnancy had significantly higher overall energy intake and saturated fat consumption compared with the referent group.

Dellava, Von Holle, Dietary supplements More than $90 \%$ of women from each eating disorder category used a dietary ..., Bulik: $2011(46)$ supplement during pregnancy at least once i.e., folic acid. There was no difference in supplement use between women with eating disorders and referent women after adjustment for sociodemographic factors. Unadjusted results showed that women with BED were less likely to consume any supplement across pregnancy, and were especially less likely to consume folic acid, vitamin D, iron, and cod-liver oil/omega 3 fatty acids.

Torgersen, Ystrom, Breastfeeding Almost all women initiated breastfeeding (98\%), with no differences between the ..., Bulik: $2010(48)$ eating disorder and referent group. The eating disorder and referent groups generally used breastfeeding as the predominant nutrition source for infants; however, mothers with BED were significantly less likely to than mothers in the referent group. Among women with all types of eating disorders, there was a significantly higher risk of ceasing breastfeeding by six months postpartum, which remained for women with AN and EDNOS-P after adjusting for covariates.

\begin{tabular}{ll}
\hline Ulman, Von Holle, & Sleeping difficulties \\
$\ldots$, Bulik: & and BED \\
$2012(54)$ &
\end{tabular}
BED before and during pregnancy was associated with higher odds of sleeping problems at 18 weeks of pregnancy. Incident BED during pregnancy was associated with higher (>10 vs. 6-9) and lower ( $<6$ vs. $6-9)$ hours of sleep in the third trimester. Women with BED before and during pregnancy reported greater dissatisfaction with sleep 18 months after childbirth.

Perrin, Von Holle, Infant growth ..., Bulik: 0-12 month weight-for-length growth rate was significantly lower among infants of $2014(55)$ women with AN, BN, BED, and EDNOS-P compared with infants of women without eating disorders. The associations were significant before and after adjustment for confounders. The hypothesis that infants of women with BN or BED would have higher growth rates than the referent women was not supported.

Zerwas, Von Holle, Infant temperament ..., Bulik: Eating disorders in pregnancy predicted greater maternal ratings of difficult infant temperament at six months of age. The associations were significant for $\mathrm{AN}, \mathrm{BN}$, BED, and EDNOS-P, odds ratios were moderate to large in magnitude, and held when confounding explanations such as greater smoking during pregnancy, greater pre-term births, and postpartum depression were adjusted for. Women with eating disorders were significantly more likely to rate their children at the most extreme level of difficult infant temperament in comparison to referent women.

\begin{tabular}{lll}
\hline $\begin{array}{l}\text { Reba-Harrelson, } \\
\text { Von Holle, } \ldots,\end{array} \quad \begin{array}{l}\text { Toddler eating } \\
\text { problems }\end{array}$ & $\begin{array}{l}\text { Toddler eating problems were rated by maternal report in MoBa and covered not } \\
\text { eating well, having stomach aches or cramps, vomiting without medical cause, and } \\
\text { Bulik: }\end{array}$ \\
$\begin{array}{ll}\text { not enjoying eating. Adjusting for child food allergy and weight status, eating } \\
\text { problems significantly differed across maternal eating disorder groups. Mothers }\end{array}$ \\
\\
with BN and BED reported greater eating problems among offspring than referent \\
mothers. The eating problems were correlated with maternally-reported maternal \\
restrictive feeding style and maternal pressure to eat.
\end{tabular}

Note: For brevity, first two and final author/s are shown. $\mathrm{AN}=$ anorexia nervosa. $\mathrm{BED}=$ binge eating disorder. $\mathrm{BN}=$ bulimia nervosa. EDNOS-P = eating disorder not otherwise specified - purging disorder. 


\section{Risk correlates}

Prior to the studies of risk correlates and risk factors within $\mathrm{MoBa}$ few investigations existed, despite interest among clinicians (i.e., 19,20). In a case series, Tiller and Treasure (20) noted common vulnerabilities in women with incident eating disorders in pregnancy, implicating partner relationship difficulties, ambivalence about motherhood, physical problems during pregnancy and labor, depression, anxiety, childhood abuse, and weight criticism. The design of Tiller and Treasure's study did not allow generalization to the population since it was based on few cases, exposures were not quantifiably measured, and there was no comparison group. MoBa analyses likewise suggested that psychosocial adversities increase susceptibility to eating disorders in pregnancy (22-24). Exposureoutcome associations observed in MoBa raise detection and diagnosis opportunities.

\section{Pregnancy, ObSTETRIC, AND BIRTh CORRELATES}

\section{Unintended pregnancies}

Unintended pregnancy is a public health concern. Approximately $22 \%$ of worldwide births are unplanned at the time of conception (25). Unintended pregnancy carries greater risks for mother and child, including higher odds of maternal mortality, maternal mental illness, child mortality, impaired child growth, child abuse, lack of and early cessation of breastfeeding, and delayed and inadequate prenatal care (26). In less developed countries, abortion practices to manage unintended pregnancies may not be accessible or may be unsafe. A MoBa investigation by our group (27) found a greater than two-fold higher rate of unintended pregnancies among women with AN compared with referent women. One possible reason for this may be that health professionals and patients assume incorrectly that pregnancy cannot occur in the context of absent or irregular menstruation leading to lower contraception use (28).

\section{Birth and obstetric outcomes}

Some community and clinical studies have suggested that eating disorders have a negative effect on birth outcomes, in actively ill mothers and those with a past history. For instance, one study found that women with a history of eating disorder hospitalization had babies with lower birth weight (7). MoBa afforded the opportunity to explore these observations on a population level. A MoBa study (29) provided evidence that eating disorders are associated with birth complications, but the range of associations was limited and patterns expected from earlier clinical studies were not replicated. Bulik et al. highlighted two explanations; firstly, clinical samples include individuals with more severe eating disorders in whom adverse outcomes may be more likely to occur. Second, the study had inadequate statistical power for the lower prevalence disorders (e.g., AN); over double the sample size would have been necessary to yield adequate statistical power, highlighting the difficulty in progressing science in this area. A recent Finnish population-based register study $(N=11,285)$ (30) compared women with lifetime eating disorders to referent women. Women with lifetime AN and BN gave birth to significantly lower weight babies, and women with BED to higher weight babies. Maternal AN was significantly associated with slow fetal growth, premature contractions, very premature birth, and perinatal death. $\mathrm{BN}$ was associated with premature contractions, resuscitations of the neonate, and a low APGAR score. Research on AN etiology in general suggests that birth complications are associated with onset of $\mathrm{AN}$, and that the birth complications are due to environmental not genetic risk factors, so may be preventable (31). Collectively, the findings suggest that women with eating disorders are at higher risk of undesirable birth and obstetric outcomes during pregnancy.

\section{Sex ratio}

An exploratory study by Bulik and colleagues (32) found indications that $\mathrm{MoBa}$ women with $\mathrm{AN}$ and $\mathrm{BN}$ had a lower sex ratio (higher proportion of females), while women with BED and EDNOS-P had a higher sex ratio (higher proportion of male births), although the differences with referent women were not statistically significant. The small differences may be due to the small sample size with eating disorders, the uncertainty in the estimates suggests that future research with larger samples is required to clarify the magnitude of the risk ratios. Norwegian sex ratio estimates for general population samples range from 1.04 to 1.06 (32). Sex ratio at birth may be affected by factors subject to disruption among women with eating disorders, such as stress, nutrition, weight, and hormonal factors (32). A lower sex ratio among mammals has been associated with maternal stress, lower weight mothers, maternal diets low in fat, and low maternal estrogen (33). These factors may be related to the small but possibly meaningful differences in sex ratio found in MoBa.

\section{Maternal Correlates}

\section{Attitudes to weight gain in pregnancy}

We examined attitudes toward weight gain among MoBa women and observed higher concern across eating disorder groups than referent women in early pregnancy (34). In an ALSPAC study (16), concern about weight gain, desire to lose weight, and believing that one had put too much weight on during pregnancy were also significantly higher among those reporting a recent or lifetime history of eating disorders. In ALSPAC, the eating disorder group was over 5 times more likely to diet at 32 weeks pregnancy. Body dissatisfaction can increase in late pregnancy among 
women with a history of restrictive dieting (35). Alternatively, some pregnant women with a history of eating disorders feel liberated from weight worries; they report appreciation of function over appearance, gaining new meaning that attenuates body concerns, and positive social commentary on their changing body (36). Finding a way to reduce concern over weight gain in early pregnancy may reduce the use of unhealthy weight control strategies.

\section{Gestational weight gain}

We investigated gestational weight gain (37) and found different patterns by eating disorder subtype relative to pregnant women without a history of eating disorders. Higher weight gain was observed in BN and BED, which is undesirable from a public health perspective as it is a potential pathway for negative maternal and fetal outcomes such as gestational diabetes, large for gestational age, fetal metabolic programming, and maternal obesity. For AN where significant underweight is a key aspect of the presentation, the higher weight gain observed may be restorative and offer protection against adverse outcomes.

\section{Pregnancy-related nausea and vomiting}

Since, in general, women with eating disorders are at increased risk of maternal and fetal complications (29, 30 ), they may be at increased risk of other difficulties in pregnancy such as pregnancy-related nausea and hyperemesis gravidarum. Given the centrality of vomiting in some eating disorder presentations and during pregnancy, a MoBa investigation by Torgersen et al. (38) examined pregnancy-related vomiting and nausea, and confirmed more difficulties among those with eating disorders.

In the general population, nausea and vomiting occur in the first trimester in up to $80 \%$ of pregnancies (39). Vomiting may in theory adversely affect appetite, hydration, and nutritional intake; adequate nutrition may already be a challenge among women with eating disorders. Animal models suggest that extracellular dehydration which occurs through vomiting in pregnancy leads to measurable increased salt appetite in offspring, which is a risk factor for hypertension and other physical problems in offspring (40). Vomiting in pregnancy has been linked to food aversions (41), among those with restricting eating disorders this is undesirable as the variety of foods tolerated by the individual may already be too narrow for health and well-being.

MoBa evidence of an association between $\mathrm{BN}$ purging type and pregnancy-related vomiting suggests that there may be common neurobiological and psychological substrates. Speculated causes of pregnancyrelated nausea and vomiting include human chorionic gonadotrophin (hCG), estrogen, gastric dysfunction, and vitamin B deficiency, but as yet, the etiology is elusive (42). Understanding the underlying substrates may lead to therapeutic advances in treatment for both conditions.

\section{Dietary intake}

Dietary intake during pregnancy influences infant health. Maternal high-fat diet consumption, such as that observed in MoBa women with BED (43), may result in fetal programming that predisposes the index child to overeating and metabolic syndrome later in life. Animal models suggest that a maternal diet higher in fat may dysregulate serotonergic pathways in the fetus that are associated with future onset of behavioral problems including anxiety (44). The energy dense diet may also lead to greater weight gain during pregnancy, posing problems for the extinction of disordered eating through induction of binge eating triggers of negative affect and body dissatisfaction. The ALSPAC cohort study (45) found that women who self-reported a lifetime eating disorder had some minor differences in macronutrient intake compared with referent women; those with lifetime AN consumed lower saturated fat and those with $\mathrm{BN}$ consumed higher polyunsaturated fat. There were, however, no differences in mineral and vitamin intakes.

Little is known about the use of dietary supplements among pregnant women with eating disorders, who are at higher risk of deficiencies due to their illness. Dellava and colleagues (46) investigated dietary supplement use during pregnancy among MoBa women with current or recent $\mathrm{AN}, \mathrm{BN}, \mathrm{BED}$, and EDNOS-P, and found similar intakes. Overall, referent and nonreferent women in MoBa did not meet the recommended micronutrient intake through diet and supplements $(43,46)$.

\section{Breastfeeding}

Breastfeeding provides numerous preventive health benefits to mother and baby and is a public health and nutrition priority. Breastfed infants are less likely to develop infectious diseases, diabetes, leukemia, gastrointestinal and respiratory diseases, and sudden infant death syndrome, and breastfeeding mothers are at lower risk of diabetes, and breast and ovarian cancer (47). Breastfeeding behaviors among women in MoBa were studied by Torgersen et al. (48), and adverse outcomes were found for women with eating disorders. There is limited other research on breastfeeding in eating disorders. A smaller population-based study in Sweden found that compared to women without eating disorders, women grouped by prior or current eating disorder were significantly more likely to have terminated breastfeeding by three months postpartum (49). A case series documented difficulties breastfeeding amongst mothers with $\mathrm{AN}$, which for some infants was associated with poor growth (50). Interestingly, the ALSPAC study found the opposite to MoBa, that women with eating disorders were significantly less likely to cease breastfeeding relative to referent women, they pointed out that this may have arisen from differences in case definition (51) or may relate to sociocultural artifacts, since fewer women in the general population in the United Kingdom (76\%) breastfed compared with Norway (98\%) and Sweden (93\%). 
Shared risks associated with eating disorders and breastfeeding difficulty, such as maternal age, maternal weight, education level, and birth outcomes may interfere with maternal breastfeeding. Cessation of breastfeeding could be due to difficulties with breast milk supply, which is dependent upon a complex cascade of hormonal and nutritional factors that may be disrupted in people with eating disorders. Breastfeeding support interventions may be a way to improve child and maternal health outcomes among those with eating disorders, particularly for AN and EDNOS-P.

\section{Sleep}

Most pregnant women report alterations in sleep, with impairment correlating with trimester of pregnancy. Disruptions in the third trimester are most common, due to abdominal discomfort, fetal movement, back pain, and hormonal changes (52). Postpartum effects on sleep are profuse, with infant sleeping and feeding practices increasing the risk of sleeping problems. Research into sleep in eating disorders has received relatively little attention. For $\mathrm{AN}$ and $\mathrm{BN}$, subjective sleep studies indicate impairments in quality of sleep while objective sleep studies find little difference in comparison with healthy controls (53).

BED and sleeping problems have not been studied to-date in the general population. A MoBa study examined sleeping problems in BED (54) and observed a positive association. An association is plausible because binge eating is associated with delayed and poorer quality sleep, and obesity increases the risk of sleeping problems and is often comorbid with BED. Potential mechanisms include appetite hormones implicated in sleeping and eating disorders, as well as stress and cortisol, although further study and replication of these findings are needed.

\section{Child CORRELATES}

\section{Infant growth}

Infant growth rate was significantly lower among infants of MoBa women with all types of eating disorder compared with referent women, although the effects were modest (55). The findings may ostensibly be related to breastfeeding difficulties $(48,56)$ and breast milk quality and quantity, which may be affected by maternal dietary restriction in the context of gestational weight gain, hormonal factors, and self-consciousness about nursing in public (55).

Greater feeding and eating difficulties have been found among one- and six-month old infants of mothers with histories of AN than referent women in the ALSPAC cohort, including slow eating and eating smaller amounts (51). However, the ALSPAC analysis found no difference in the 0-9 month growth rate of infants of mothers with a history of $\mathrm{AN}$, and a larger 0-9 month growth rate than referents among infants of women with a history of BN. Possibly, a longer time span than observed in ALSPAC is needed to observe an impact of AN history on infant growth, or there was insufficient statistical power. For infants of $\mathrm{MoBa}$ mothers with a history of $\mathrm{BN}$, growth accelerated beyond the referent group between 4 and 8 months, but leveled to 12 months, meaning that the differences observed in ALSPAC at 9 months may have washed out in the MoBa observation period of 12 months (55).

\section{Infant temperament}

In $\mathrm{MoBa}$, eating disorders in pregnancy predicted greater maternal ratings of difficult infant temperament at six months of age (57). Infant temperament has well-established links with maternal psychiatric status. Fussy or difficult infant temperament serves as a marker for later difficulty with emotion regulation, and temperament may have bidirectional relations with parental attachment (58). The use of maternal report of child temperament rather than a direct observational measure was a limitation of the $\mathrm{MoBa}$ paper. The explanation for the association between difficult infant temperament and maternal eating disorder status remains unclear. Mothers with eating disorders may just perceive their infants as more difficult. The social information processing biases observed in eating disorders may lead mothers to interpret their infants' behavior as more emotionally negative (59). Mothers with eating disorders also tend to struggle with greater anxiety. Anxiety, in turn, predicts report of perceived "difficult" infant temperament at six months, independent of maternal depression (60).

Possibly, infants of mothers with eating disorders could objectively display more difficult temperaments. Eating disorders tend to run in families due to shared genetic susceptibilities, and difficult infant temperament may signal the early expression of risk genes and a predisposition towards later emotional reactivity and anxiety (61). Individual differences in the intrauterine environment of mothers with eating disorders could play a role. Maternal anxiety and cortisol during the prenatal period predict greater cortisol stress response in children and could reflect an early emergence of a prenatally programmed stress response. Other research suggests that birth complications such as neonatal dysmaturity influence the development of offspring temperament (62).

\section{Toddler eating problems}

Reba-Harrelson et al. (63) used MoBa data to investigate the relationship between maternal eating disorders and child eating problems at 32 months, finding significantly greater problems in offspring of mothers with $\mathrm{BN}$ and BED. Medical concern has been expressed that parents with eating disorders may inadequately nourish their children, by underfeeding (6) or overfeeding (51). Women with eating disorders may find it harder to distinguish between normal versus abnormal eating practices, may have difficulty keeping food items available and accessible because of a fear of binge eating, and may find being around food a 
stressful part of daily experience. A small observational study found that even after controlling for child's birth weight and mother's height, at 12 months the infants of women with eating disorders weighed less than their healthy counterparts (64). The MoBa data will be informative for examining how child eating outcomes correlate with growth and development trajectories. The ALSPAC group (51) similarly found that eating problems were greater at nine months for infants of mothers with eating disorders and a referent general psychiatric group, in comparison with a healthy control group, and that maternal anxiety and depression mediated the effect of lifetime eating disorder on infant eating problems (65).

\section{FUTURE DIRECTIONS}

Although our systematic inquiries into eating disorders and pregnancy in $\mathrm{MoBa}$ have yielded considerable new knowledge, many important questions remain. The prevalence of eating disorders in the first trimester of pregnancy was on par with general population prevalence in females. An area for future research not currently evaluable within $\mathrm{MoBa}$ is to assess prevalence by trimester, since differences have been observed for other psychiatric disorders, such as higher depression risk in the second and third trimesters (66). The incidence rate of BED in pregnancy needs further consideration; it is unclear to what extent the measurement of binge eating in pregnancy is capturing enduring pathology. The investigation of overlap may also prove valuable, for there may be shared neural mechanisms underlying patterns of dysregulated eating (67). Eating disorder status and risk correlates have not yet been examined in the context of postpartum eating disorder persistence or other illness courses. As lifetime eating disorder status is now available for $\mathrm{MoBa}$ mothers, it will be important to review the unique association of eating disorders with birth and other maternal and child health outcomes. The MoBa study has shed light on some aspects of breastfeeding practices in women with eating disorders, but not others. A case report has suggested that women with eating disorders could use breast milk expression as a purgatory method (68) or prolong breastfeeding as a means to control body weight (20). MoBa is unable to address this concerning trend. Future data from $\mathrm{MoBa}$ and ALSPAC on infant and child growth among index children of mothers with eating disorders will be important for understanding the weight status and chronic disease risk at a population level of offspring with maternal exposure to eating disorders. The observed predisposition towards negative, difficult, and fussy emotionality in offspring of mothers with eating disorders might evoke compromised maternal caregiving, which in turn could lead to a self-perpetuating cycle of risk. Continuing to examine the development of children of mothers with eating disorders could interrupt this potential cycle in the future.
Two broad research directions within the planned research program are reproducibility, and a better understanding of the contribution of genes and environment to outcomes. Reproducibility is one of the most central parts of science, but replication and validation studies rarely appear in peer-reviewed journals. Now that $\mathrm{MoBa}$ recruitment is completed, we plan to validate our earlier work and have completed two validation studies to-date $(15,24)$. Eating disorders and milder eating phenotypes are known to aggregate in families. Women with eating disorders may transmit genetically encoded traits and behaviors in offspring or exert other influences on the child's environment that affect normal eating and predispose to future eating disorders. Understanding the role of family eating disorder and phenotype history, fathers included, may prove important. We would like to make use of the biobank and explore genetic effects, environmental effects, gene-environment co-action, and epigenetic factors on risk for eating disorders in the $\mathrm{MoBa}$ children.

\section{LIMITATIONS AND CHALLENGES}

There are important limitations and challenges to the MoBa studies on eating disorders; firstly, the methodology used to diagnose eating disorders is based on selfreport. Psychiatric diagnosis with self-report methodology is common in epidemiological research due to the practical difficulties and cost of administering structured diagnostic interviews to a large group of people $(100,000$ in the case of MoBa). Yet discordance between self-report and expert-ratings can arise, for example on the criteria for a binge episode in eating disorders. Self-report methodology has convergence with expert psychiatric diagnosis for well-defined eating disorders, but underestimates overall prevalence since DSM nomenclature has not defined other presentations well ("unspecified eating disorders"). The studies on risk factors have limitations that need to be considered. Causality from exposure-to-outcome cannot be inferred and in the context of limited statistical power only univariate models were considered, yet correlated, complex relationships among risk factors are inevitable. There is the possibility of self-selection bias in $\mathrm{MoBa}$ - mothers participating in MoBa have healthier lifestyle patterns and higher socioeconomic status, which may affect maternal and child outcomes. Possible selection bias may be particularly important to consider for women with eating disorders. There are no available data to assess the degree or direction of selection bias for women with eating disorders, in addition, little is known about how representative women with eating disorders who become pregnant are of the general female eating disorder population.

A challenge for analysts working with MoBa data is the inability to link individuals across dataset versions because individual identification numbers change across versions. This has made validation studies (such 
as split-sample comparisons) challenging, since the sample used in the initial study cannot be perfectly identified in a later $\mathrm{MoBa}$ dataset version.

\section{FinAl CONCLUSION}

MoBa evidence suggests that eating disorders affect $\sim 5 \%$ of pregnant women $(13,15)$. We have observed that pregnancy may be a high-risk period for onset of BED, but positively, may be a window of remission for $\mathrm{BN}(13,15)$. Women with lifetime and current psychosocial adversities are most at risk of developing incident eating disorders or if they have eating disorders, for having a poor prognosis during pregnancy $(22,23)$. MoBa research has shown that eating disorders amplify the odds of negative birth and obstetric outcomes, although these have not been as extreme as thought on the basis of earlier clinical literature (29). This may be because MoBa better represents eating disorder cases in the community, rather than only the proportion (approximately 40\%) who seek treatment. Eating disorders in pregnancy are associated with negative maternal outcomes, relative to women in the general community: women with BED have more sleeping problems (54), women with BN-purging subtype experience more nausea and pregnancy-related vomiting (38), women with BN and BED have excessive weight gain (37), and women with any eating disorder experience more distress about weight gain (34). Maternal eating disorders were associated with negative outcomes for MoBa children, including greater eating problems (63) and difficult infant temperament (57), although temperament may be attributable to the mother's own level of distress. A higher fat and energy-dense diet predominates among pregnant wo- men with BED (43). This may preprogram metabolism and neurotransmission in offspring, although offspring growth rates in infancy were not higher (55). Another important finding from MoBa research has been the higher prevalence of unintended pregnancies among women with AN pre-pregnancy relative to women in the general population (27).

MoBa has provided an unprecedented opportunity to investigate eating disorders in pregnancy at a population level. The rich and comprehensive data available have allowed a diverse set of research questions to be investigated. MoBa has been critical in advancing knowledge; in response, health professionals and policy makers should aim to increase their understanding of eating disorders in pregnancy to optimize outcomes for mother, child, and the broader community.

\section{ACKNOWLEDGEMENTS AND DECLARATION OF INTEREST}

We are grateful to all the participating families in Norway who take part in this ongoing cohort study.

This research was supported by R01HD047186 Eating Disorders and Pregnancy Outcome in 100,000 Births (Bulik: PI); F31 HD057719 Mothers with eating disorders and their children maternal feeding \& child eating (Reba-Harrelson: PI); K01 MH100435 Developmental Psychopathology of Restrictive and Binge Eating in Childhood (Zerwas: PI); a Norwegian Marshall Fund Research Grant to Dr. RebaHarrelson; and T32MH076694 (Bulik: PI). The Norwegian Mother and Child Cohort Study is supported by the Norwegian Ministry of Health and the Ministry of Education and Research, NIH/NIEHS (contract no NO-ES-75558), NIH/NINDS (grant no.1 UO1 NS 047537-01 and grant no. 2 UO1 NS 047537-06A1), and the Norwegian Research Council/FUGE (grant no. 151918/S10).

Dr. Bulik is a Consultant for Shire Pharmaceuticals. Other authors report no conflicts of interest.

\section{REFERENCES}

*References marked with an asterisk identify MoBa studies on eating disorders in pregnancy

1. American Psychiatric Association. Diagnostic and statistical manual of mental disorders. 5th ed. Washington, DC: American Psychiatric Association; 2013.

2. Götestam KG, Agras WS. General population-based epidemiological study of eating disorders in Norway. International Journal of Eating Disorders 1995;18(2):119-26.

3. Pomeroy C, Mitchell JE. Medical complications of anorexia nervosa and bulimia nervosa. Eating disorders and obesity: A comprehensive handbook 2002;2:278-85.

4. Arcelus J, Mitchell AJ, Wales J, Nielsen S. Mortality rates in patients with anorexia nervosa and other eating disorders: a meta-analysis of 36 studies. Archives of General Psychiatry 2011;68(7):724-31.

5. Hudson JI, Hiripi E, Pope Jr HG, Kessler RC. The prevalence and correlates of eating disorders in the National Comorbidity Survey Replication. Biological Psychiatry 2007;61(3):348-58.

6. Waugh E, Bulik CM. Offspring of women with eating disorders. International Journal of Eating Disorders 1999;25(2):123-33.

7. Sollid CP, Wisborg K, Hjort J, Secher NJ. Eating disorder that was diagnosed before pregnancy and pregnancy outcome. American Journal of Obstetrics and Gynecology 2004;190(1):206-10.

8. Lemberg R, Phillips J. The impact of pregnancy on anorexia nervosa and bulimia. International Journal of Eating Disorders 1989;8(3):285-95.

9. Golding J, Pembrey M, Jones R. ALSPAC - the Avon Longitudinal Study of Parents and Children. I. Study methodology. Paediatric and Perinatal Epidemiology 2001;15(1):74-87. 
10. Favaro A, Tenconi E, Santonastaso P. Perinatal factors and the risk of developing anorexia nervosa and bulimia nervosa. Archives of General Psychiatry 2006;63(1):82-8.

11. Magnus P, Irgens LM, Haug K, Nystad W, Skjærven R, Stoltenberg C. Cohort profile: the Norwegian mother and child cohort study (MoBa). International Journal of Epidemiology 2006;35(5):1146-50.

12. Association AP. DSM-IV-TR: Diagnostic and statistical manual of mental disorders, text revision: American Psychiatric Association; 2000.

13. *Bulik CM, Von Holle A, Hamer R, Knoph Berg C, Torgersen L, Magnus P, et al. Patterns of remission, continuation and incidence of broadly defined eating disorders during early pregnancy in the Norwegian Mother and Child Cohort Study (MoBa). Psychological medicine 2007;37(8):1109-18.

14. American Psychiatric Association. Diagnostic and statistical manual of mental disorders. 1994.

15. *Watson HJ, Von Holle A, Hamer RM, Knoph Berg C, Torgersen L, Magnus P, et al. Remission, continuation and incidence of eating disorders during early pregnancy: A validation study in a population-based birth cohort. Psychological medicine 2012:1-12.

16. Micali N, Treasure J, Simonoff E. Eating disorders symptoms in pregnancy: a longitudinal study of women with recent and past eating disorders and obesity. Journal of Psychosomatic Research 2007;63(3):297-303.

17. Easter A, Bye A, Taborelli E, Corfield F, Schmidt U, Treasure J, et al. Recognising the symptoms: how common are eating disorders in pregnancy? European Eating Disorders Review 2013;21(4):340-4.

18. *Knoph C, Von Holle A, Zerwas S, Torgersen L, Tambs K, Stoltenberg C, et al. Course and predictors of maternal eating disorders in the postpartum period. International Journal of Eating Disorders 2013;46(4): 355-68.

19. Blais MA, Becker AE, Burwell RA, Flores AT, Nussbaum KM, Greenwood DN, et al. Pregnancy: Outcome and impact on symptomatology in a cohort of eating-disordered women. International Journal of Eating Disorders 2000;27(2):140-9.

20. Tiller J, Treasure J. Eating disorders precipitated by pregnancy. European Eating Disorders Review 1998;6(3):178-87.

21. Lacey JH, Smith G. Bulimia nervosa. The impact of pregnancy on mother and baby. British Journal of Psychiatry 1987;150(6):777-81.

22. *Knoph Berg C, Bulik CM, Von Holle A, Torgersen L, Hamer R, Sullivan P, et al. Psychosocial factors associated with broadly defined bulimia nervosa during early pregnancy: Findings from the Norwegian Mother and Child Cohort Study. The Australian and New Zealand journal of psychiatry 2008;42:396-404.

23. *Knoph Berg C, Torgersen L, Von Holle A, Hamer RM, Bulik CM, Reichborn - Kjennerud T. Factors associated with binge eating disorder in pregnancy. International Journal of Eating Disorders 2011;44(2):124-33.

24. *Watson HJ, Von Holle A, Knoph C, Hamer RM, Torgersen L, Reichborn-Kjennerud T, et al. Psychosocial factors associated with bulimia nervosa during pregnancy: An internal validation study. International Journal of Eating Disorders in press.

25. Singh S, Sedgh G, Hussain R. Unintended pregnancy: worldwide levels, trends, and outcomes. Studies in Family Planning 2010;41(4):241-50.

26. Gipson JD, Koenig MA, Hindin MJ. The effects of unintended pregnancy on infant, child, and parental health: a review of the literature. Studies in family planning 2008;39(1):18-38.

27. *Bulik CM, Hoffman ER, Von Holle A, Torgersen L, Stoltenberg C, Reichborn-Kjennerud T. Unplanned pregnancy in women with anorexia nervosa. Obstetrics and Gynecology 2010;116(5):1136-40.

28. Sawyer SM, Tully M-AM, Colin AA. Reproductive and sexual health in males with cystic fibrosis: a case for health professional education and training. Journal of Adolescent Health 2001;28(1):36-40.

29. *Bulik CM, Von Holle A, Siega-Riz AM, Torgersen L, Lie KK, Hamer RM, et al. Birth outcomes in women with eating disorders in the Norwegian Mother and Child cohort study (MoBa). International Journal of Eating Disorders 2009;42(1):9-18.

30. Linna MS, Raevuori A, Haukka J, Suvisaari JM, Suokas JT, Gissler M. Pregnancy, obstetric and perinatal health outcomes in eating disorders. American Journal of Obstetrics and Gynecology 2014.

31. Favaro A, Tenconi E, Bosello R, Degortes D, Santonastaso P. Perinatal complications in unaffected sisters of anorexia nervosa patients: testing a covariation model between genetic and environmental factors. European Archives of Psychiatry and Clinical Neuroscience 2011;261(6):391-6.

32. *Bulik CM, Von Holle A, Gendall K, Lie KK, Hoffman E, Mo X, et al. Maternal eating disorders influence sex ratio at birth. Acta Obstetricia et Gynecologica Scandinavica 2008;87(9):979-81.

33. Rosenfeld CS, Roberts RM. Maternal diet and other factors affecting offspring sex ratio: a review. Biology of Reproduction 2004;71(4):1063-70.

34. *Swann RA, Von Holle A, Torgersen L, Gendall K, Reichborn-Kjennerud T, Bulik CM. Attitudes toward weight gain during pregnancy: results from the Norwegian mother and child cohort study (MoBa). International Journal of Eating Disorders 2009;42(5):394-401. 
35. Rocco PL, Orbitello B, Perini L, Pera V, Ciano RP, Balestrieri M. Effects of pregnancy on eating attitudes and disorders: a prospective study. Journal of Psychosomatic Research 2005;59(3):175-9.

36. Clark, Skouteris H, Wertheim EH, Paxton SJ, Milgrom J. My baby body: A qualitative insight into women's body-related experiences and mood during pregnancy and the postpartum. Journal of Reproductive and Infant Psychology 2009;27(4):330-45.

37. *Siega-Riz AM, Von Holle A, Haugen M, Meltzer HM, Hamer R, Torgersen L, et al. Gestational weight gain of women with eating disorders in the Norwegian pregnancy cohort. International Journal of Eating Disorders 2011;44(5):428-34.

38. *Torgersen L, Von Holle A, Reichborn-Kjennerud T, Berg CK, Hamer R, Sullivan P, et al. Nausea and vomiting of pregnancy in women with bulimia nervosa and eating disorders not otherwise specified. International Journal of Eating Disorders 2008;41(8):722-7.

39. Gadsby R, Barnie-Adshead AM, Jagger C. A prospective study of nausea and vomiting during pregnancy. British Journal of General Practice 1993;43(371):245.

40. Nicolaidis S, Galaverna O, Metzler CH. Extracellular dehydration during pregnancy increases salt appetite of offspring. American Journal of Physiology-Regulatory, Integrative and Comparative Physiology 1990;258(1): R281-R3.

41. Bayley TM, Dye L, Jones S, DeBono M, Hill AJ. Food cravings and aversions during pregnancy: relationships with nausea and vomiting. Appetite 2002;38(1):45-51.

42. Davis M. Nausea and vomiting of pregnancy: an evidence-based review. Journal of Perinatal and Neonatal Nursing 2004;18(4):312-28.

43. *Siega-Riz AM, Haugen M, Meltzer HM, Von Holle A, Hamer R, Torgersen L, et al. Nutrient and food group intakes of women with and without bulimia nervosa and binge eating disorder during pregnancy. American journal of clinical nutrition 2008;87(5):1346-55.

44. Sullivan EL, Grayson B, Takahashi D, Robertson N, Maier A, Bethea CL, et al. Chronic consumption of a high-fat diet during pregnancy causes perturbations in the serotonergic system and increased anxiety-like behavior in nonhuman primate offspring. Journal of Neuroscience 2010;30(10):3826-30.

45. Micali N, Northstone K, Emmett P, Naumann U, Treasure JL. Nutritional intake and dietary patterns in pregnancy: a longitudinal study of women with lifetime eating disorders. British Journal of Nutrition 2012;108 (11):2093-9.

46. *Dellava JE, Von Holle A, Torgersen L, Reichborn-Kjennerud T, Haugen M, Meltzer HM, et al. Dietary supplement use immediately before and during pregnancy in Norwegian women with eating disorders. International Journal of Eating Disorders 2011;44(4):325-32.

47. Ip S, Chung M, Raman G, Chew P, Magula N, DeVine D, et al. Breastfeeding and maternal and infant health outcomes in developed countries. Rockville, Maryland: Agency for Healthcare Research and Quality, 2007.

48. *Torgersen L, Ystrom E, Haugen M, Meltzer HM, Von Holle A, Berg CK, et al. Breastfeeding practice in mothers with eating disorders. Maternal \& Child Nutrition 2010;6(3):243-52.

49. Larsson G, Andersson-Ellström A. Experiences of pregnancy-related body shape changes and of breastfeeding in women with a history of eating disorders. European Eating Disorders Review 2003;11(2):116-24.

50. Treasure JL, Russell GFM. Intrauterine growth and neonatal weight gain in babies of women with anorexia nervosa. British Medical Journal 1988;296:1038.

51. Micali N, Simonoff E, Treasure J. Infant feeding and weight in the first year of life in babies of women with eating disorders. Journal of Pediatrics 2009;154(1):55-60. e1.

52. Santiago JR, Nolledo MS, Kinzler W, Santiago TV. Sleep and sleep disorders in pregnancy. Annals of Internal Medicine 2001;134(5):396-408.

53. Manber R, Haynes T, Siebern AT. Sleep and psychiatric disorders. In: Morin CM, Espie CA, editors. The Oxford Handbook of Sleep and Sleep Disorders. Oxford: Oxford University Press; 2012. p. 471-501.

54. *Ulman TF, Von Holle A, Torgersen L, Stoltenberg C, Reichborn-Kjennerud T, Bulik CM. Sleep disturbances and binge eating disorder symptoms during and after pregnancy. Sleep 2012;35(10):1403.

55. *Perrin EM, Von Holle A, Zerwas S, Skinner AC, Reba - Harrelson L, Hamer RM, et al. Weight-for-length trajectories in the first year of life in children of mothers with eating disorders in a large Norwegian cohort. International Journal of Eating Disorders 2014.

56. Larsson G, Andersson - Ellström A. Experiences of pregnancy-related body shape changes and of breastfeeding in women with a history of eating disorders. European Eating Disorders Review 2003;11(2):116-24.

57. *Zerwas S, Von Holle A, Torgersen L, Reichborn-Kjennerud T, Stoltenberg C, Bulik CM. Maternal eating disorders and infant temperament: findings from the Norwegian mother and child cohort study. International Journal of Eating Disorders 2012;45(4):546-55.

58. Kochanska G, Friesenborg AE, Lange LA, Martel MM, Kochanska G. Parents' personality and infants' temperament as contributors to their emerging relationship. Journal of Personality and Social Psychology 2004;86(5):744-59. 
59. Harrison A, Tchanturia K, Treasure J. Attentional bias, emotion recognition, and emotion regulation in anorexia: State or trait? Biological Psychiatry 2010;68(8):755-61.

60. Austin MP, Hadzi-Pavlovic D, Leader L, Saint K, Parker G. Maternal trait anxiety, depression and life event stress in pregnancy: relationships with infant temperament. Early Human Development 2005;81(2):183-90.

61. Bulik C, Sullivan P, Wade T, Kendler K. Twin studies of eating disorders: a review. International Journal of Eating Disorders 2000;27:1-20.

62. Favaro A, Tenconi E, Santonastaso P. The relationship between obstetric complications and temperament in eating disorders: a mediation hypothesis. Psychosomatic Medicine 2008;70(3):372-7.

63. *Reba-Harrelson L, Von Holle A, Hamer RM, Torgersen L, Reichborn-Kjennerud T, Bulik CM. Patterns of maternal feeding and child eating associated with eating disorders in the Norwegian Mother and Child Cohort Study (MoBa). Eating behaviors 2010;11:54-61.

64. Stein A, Woolley H, Cooper SD, Fairburn CG. An observational study of mothers with eating disorders and their infants. Journal of Child Psychology and Psychiatry 1994;35(4):733-48.

65. Micali N, Simonoff E, Stahl D, Treasure J. Maternal eating disorders and infant feeding difficulties: maternal and child mediators in a longitudinal general population study. Journal of Child Psychology and Psychiatry 2011;52(7):800-7.

66. Bennett HA, Einarson A, Taddio A, Koren G, Einarson TR. Prevalence of depression during pregnancy: systematic review. Obstetrics and Gynecology 2004;103(4):698-709.

67. Mercer ME, Holder MD. Food cravings, endogenous opioid peptides, and food intake: a review. Appetite 1997;29(3):325-52.

68. Elran-Barak R, Zubery E, Steiner E. Can breast milk expression act as a compensation behavior in bulimia nervosa? Case report and literature review. 2014 International Conference on Eating Disorders; New York, United States. 2014. 